\title{
A genetic variant near olfactory receptor genes influences cilantro preference
}

\author{
Nicholas Eriksson*, Shirley Wu, Chuong B Do, Amy K Kiefer, Joyce Y Tung, Joanna L Mountain, \\ David A Hinds and Uta Francke
}

\begin{abstract}
Background: The leaves of the Coriandrum sativum plant, known as cilantro or coriander, are widely used in many cuisines around the world. However, far from being a benign culinary herb, cilantro can be polarizing —-many people love it while others claim that it tastes or smells foul, often like soap or dirt. This soapy or pungent aroma is largely attributed to several aldehydes present in cilantro. Cilantro preference is suspected to have a genetic component, yet to date nothing is known about specific mechanisms.
\end{abstract}

Results: Here, we present the results of a genome-wide association study among 14,604 participants of European ancestry who reported whether cilantro tasted soapy, with replication in a distinct set of 11,851 participants who declared whether they liked cilantro. We find a single-nucleotide polymorphism (SNP) significantly associated with soapy-taste detection that is confirmed in the cilantro preference group. This SNP, rs72921001 ( $p=6.4 \times 10^{-9}$, odds ratio 0.81 per $A$ allele), lies within a cluster of olfactory receptor genes on chromosome 11 . Among these olfactory receptor genes is $O R 6 A 2$, which has a high binding specificity for several of the aldehydes that give cilantro its characteristic odor. We also estimate the heritability of cilantro soapy-taste detection in our cohort, showing that the heritability tagged by common SNPs is low, about 0.087 .

Conclusions: These results confirm that there is a genetic component to cilantro taste perception and suggest that cilantro dislike may stem from genetic variants in olfactory receptors. We propose that one of a cluster of olfactory receptor genes, perhaps $O R 6 A 2$, may be the olfactory receptor that contributes to the detection of a soapy smell from cilantro in European populations.

Keywords: Cilantro, Coriander, Olfactory receptor, Genetics of taste and smell

\section{Background}

The Coriandrum sativum plant has been cultivated since at least the second millennium BCE [1]. Its fruits (commonly called coriander seeds) and leaves (called cilantro or coriander) are important components of many cuisines. In particular, South Asian cuisines use both the leaves and the seeds prominently, and Latin American food often incorporates the leaves.

The desirability of cilantro has been debated for centuries. Pliny claimed that coriander had important medicinal properties: 'vis magna ad refrigerandos ardores viridi' ('while green, it is possessed of very cooling and refreshing properties') [2]. The Romans used the leaves and seeds in many dishes, including moretum (a herb, cheese, and

*Correspondence: nick@23andme.com

23andMe, Inc., Mountain View, CA, 94043, USA garlic spread similar to today's pesto) [3]; the Mandarin word for cilantro, 香菜 (xiāngcài), literally means 'fragrant greens.' However, the leaves in particular have long inspired passionate hatred as well, e.g., John Gerard called it a 'very stinking herbe' with leaves of 'venemous quality' $[4,5]$.

It is not known why cilantro is so differentially perceived. The proportion of people who dislike cilantro varies widely by ancestry [6]; however, it is not clear to what extent this may be explained by differences in environmental factors, such as frequency of exposure. In a twin study, the heritability of cilantro dislike has been estimated as 0.38 (confidence interval (CI) $0.22-0.52$ ) for odor and 0.52 (CI 0.38-0.63) for flavor [7].

The smell of cilantro is often described as pungent or soapy. It is suspected, although not proven, that cilantro dislike is largely driven by the odor rather than the taste. 
Table 1 Summary of the cohorts used in the analysis

\begin{tabular}{lccc}
\hline & N & Proportion female & Age (SD) \\
\hline Tastes soapy & 1,994 & 0.566 & $49.0(15.0)$ \\
Does not taste soapy & 12,610 & 0.489 & $48.3(15.2)$ \\
Total & 14,604 & 0.500 & $48.4(15.2)$ \\
Dislikes cilantro & 3,181 & 0.487 & $47.1(16.6)$ \\
Likes cilantro & 8,906 & 0.420 & $43.8(14.5)$ \\
Total & 12,087 & 0.438 & $44.7(15.1)$ \\
\hline
\end{tabular}

The key aroma components in cilantro consist of various aldehydes, in particular (E)-2-alkenals and $n$-aldehydes $[8,9]$. The unsaturated aldehydes (mostly decanal and dodecanal) in cilantro are described as fruity, green, and pungent; the (E)-2-alkenals (mostly (E)-2-decenal and (E)-2-dodecenal) as soapy, fatty, 'like cilantro', or pungent $[8,9]$.

Several families of genes are important for taste and smell. The TAS1R and TAS2R families form sweet, umami, and bitter taste receptors [10,11]. The olfactory receptor family contains about 400 functional genes in the human genome. Each receptor binds to a set of chemicals, enabling one to recognize specific odorants or tastants. Genetic differences in many of these receptors are known to play a role in how we perceive tastes and smells [12-15].

\section{Results and discussion}

Here, we report on a genome-wide association study (GWAS) of cilantro soapy-taste detection. Briefly, the GWAS was conducted in 14,604 unrelated participants of primarily European ancestry who responded to an online questionnaire asking whether they thought cilantro tasted like soap (Table 1). Two single-nucleotide polymorphisms (SNPs) were genome-wide significant $(p<$ $5 \times 10^{-8}$ ) in this population. One SNP, in a cluster of olfactory receptors, replicated in a non-overlapping group of 11,851 participants (again, unrelated and of primarily European ancestry) who reported whether they liked or disliked cilantro (see the 'Methods' section for full details). Figure 1 shows $p$ values across the whole genome; Figure 2 shows $p$ values near the most significant associations. A quantile-quantile plot (Additional file 1$)$ shows little $(\lambda=$ 1.007) global inflation of $p$ values. Index SNPs with $p$ values under $10^{-6}$ are shown in Table 2 (along with replication $p$ values); all SNPs with $p$ values under $10^{-4}$ are shown in Additional file 2.

We found one significant association for cilantro soapytaste that was confirmed in the cilantro preference population. The SNP rs72921001 $\left(p_{\text {discovery }}=6.4 \times 10^{-9}\right.$, odds ratio $\left.(\mathrm{OR})=0.81, p_{\text {repl }}=0.0057\right)$ lies on chromosome 11 within a cluster of eight olfactory receptor genes: OR2AG2, OR2AG1, OR6A2, OR10A5, OR10A2, OR1OA4, OR2D2, and OR2D3. The C allele is associated with both detecting a soapy smell and disliking cilantro. Of the olfactory receptors encoded in this region, OR6A2 appears to be the most promising candidate underlying the association with cilantro odor detection. It is one of the most studied olfactory receptors (often as the homologous olfactory receptor I7 in rats) [16-19]. A wide range of odorants have been found to activate this receptor, all of which are aldehydes [17]. Among the unsaturated aldehydes, octanal binds best to rat I7 [18]; however, compounds ranging from heptanal to undecanal also bind to this receptor [17]. Several singly unsaturated $n$-aldehydes also show high affinity, including (E)-2-decenal [17]. These aldehydes include several of those playing a key role in cilantro aroma, such as decanal and (E)-2-decenal. Thus, this gene is particularly interesting as a candidate for cilantro odor detection. The index SNP is also in high LD $\left(r^{2}>0.9\right)$ with three non-synonymous SNPs in OR10A2, namely rs3930075, rs10839631, and rs7926083 (H43R, H207R, and K258T, respectively). Thus, OR10A2 may also be a reasonable candidate gene in this region.

The second significant association, with rs78503206 $\left(p_{\text {discovery }}=3.2 \times 10^{-8}, \mathrm{OR}=0.68, p_{\text {repl }}=0.49\right)$, lies in an intron of the gene $S N X 9$ (sorting nexin-9; see Figure 2). $S N X 9$ encodes a multifunctional protein involved in intracellular trafficking and membrane remodeling during endocytosis [20]. It has no known function in taste or smell and did not show association with liking cilantro in the replication population. This SNP is located about $80 \mathrm{~kb}$ upstream of $S Y N J 2$, an inositol 5-phosphatase thought to be involved in membrane trafficking and signal

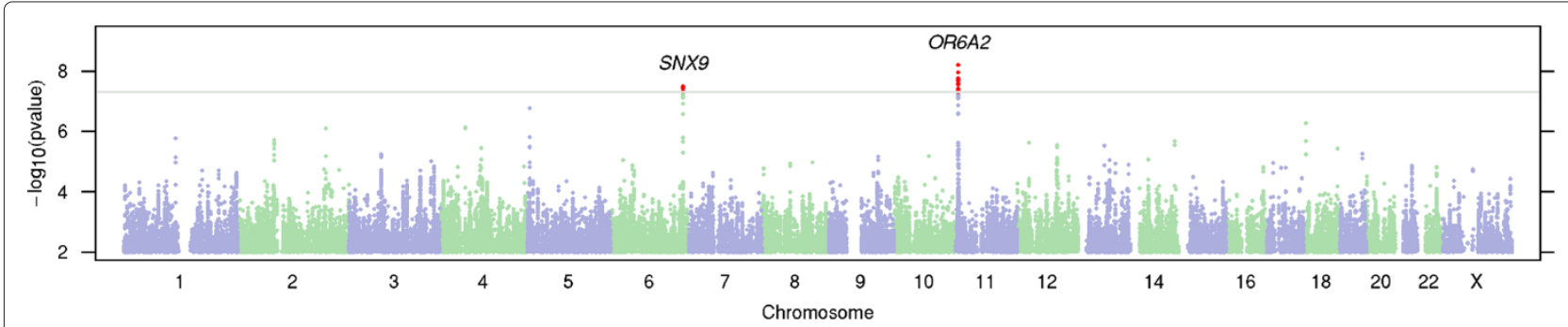

Figure 1 Manhattan plot of association with cilantro soapy-taste. Negative $\log _{10} p$ values across all SNPS tested. SNPS shown in red are genome-wide significant $\left(p<5 \times 10^{-8}\right)$. Regions are named with the postulated candidate gene. 


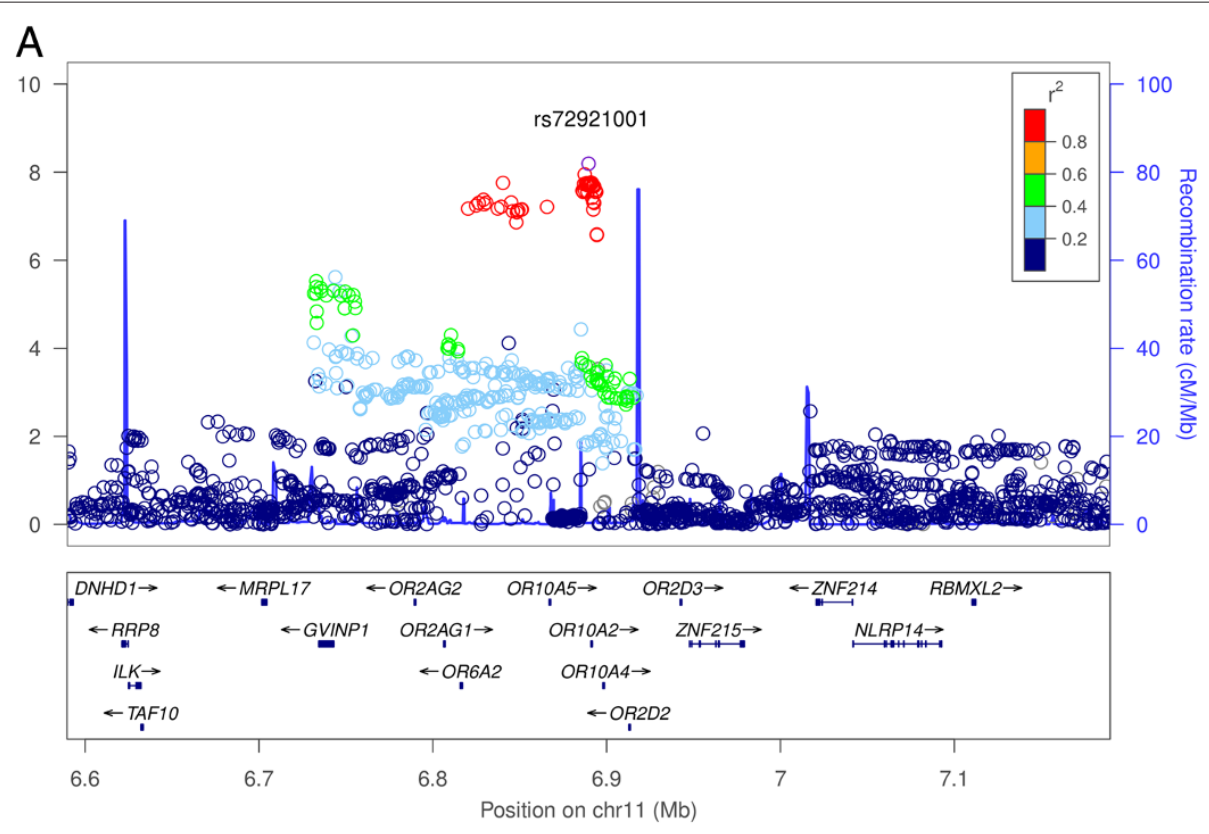

B

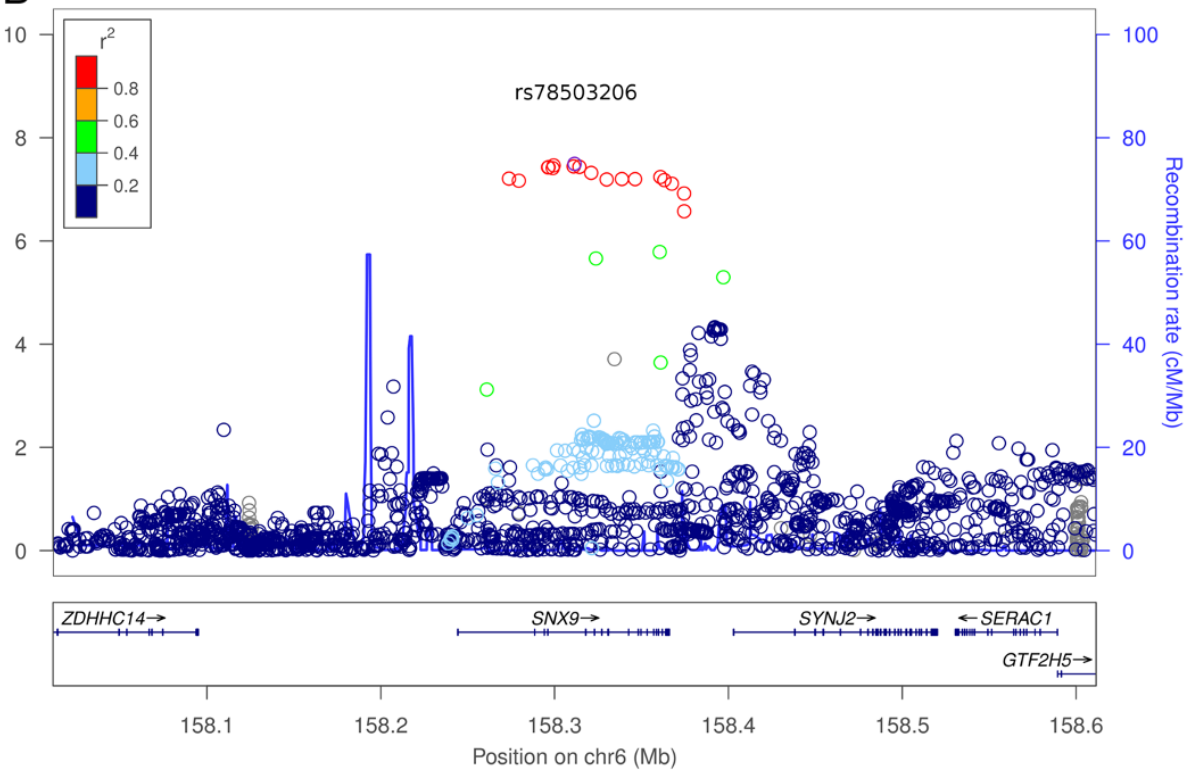

Figure 2 Associations with cilantro soapy-taste near rs72921001 (A) and rs78503206 (B). Negative $\log _{10} p$ values for association (left axis) with recombination rate (right axis). Colors depict the squared correlation $\left(r^{2}\right)$ of each SNP with the most associated SNP ((A) rs72921001 and (B) rs78503206, shown in purple). Gray indicates SNPs for which $r^{2}$ information was missing.

transduction pathways. In candidate gene studies, SYNJ2 SNPs were found to be associated with agreeableness and symptoms of depression in the elderly [21] and with cognitive abilities [22]. In mice, a Synj2 mutation causes recessive non-syndromic hearing loss [23]. Given recent evidence that the perception of flavor may be influenced by multiple sensory inputs ( $c f$. [24,25]), we cannot exclude the SYNJ2-linked SNP as conveying a biologically meaningful association. While this SNP may be a false positive, it could also be the case that this SNP is associated only with detecting a soapy smell in cilantro (and not in liking cilantro). In addition, we were unable to replicate the SNPs that were found to be nominally significant for cilantro dislike in [26] (we saw $p$ values in the GWAS of 0.53, 0.41 , and 0.53 for rs11988795, rs1524600, and rs10772397, respectively).

We have used two slightly different phenotypes in our discovery and replication, soapy-taste detection and 
Table 2 Index SNPs for regions with $p<10^{-6}$ for cilantro soapy-taste

\begin{tabular}{lccccccccc}
\hline SNP & Chromosome & Position & Gene & Allele & MAF & $\boldsymbol{r}^{2}$ & $\boldsymbol{p}_{\text {discovery }}$ & $\boldsymbol{p}_{\text {repl }}$ & OR (Cl) \\
\hline rs72921001 & 11 & $6,889,648$ & OR6A2 & C/A & 0.364 & 0.969 & $6.4 \times 10^{-9}$ & 0.0057 & $0.809(0.753-0.870)$ \\
rs78503206 & 6 & $158,311,499$ & SNX9 & C/T & 0.077 & 0.980 & $3.2 \times 10^{-8}$ & 0.49 & $0.679(0.588-0.784)$ \\
chr5:4883483 & 5 & $4,883,483$ & ADAMTS16 & C/T & 0.032 & 0.885 & $1.7 \times 10^{-7}$ & 0.51 & $0.526(0.405-0.683)$ \\
rs7227945 & 18 & $4,251,279$ & DLGAP1/LOC642597 & T/G & 0.055 & 0.920 & $5.3 \times 10^{-7}$ & 0.96 & $1.447(1.258-1.663)$ \\
rs6554267 & 4 & $56,158,891$ & KDR/SRD5A3 & T/G & 0.019 & 0.651 & $7.4 \times 10^{-7}$ & 0.85 & $1.975(1.529-2.549)$ \\
rs13412810 & 2 & $192,420,461$ & MYO1B/OBFC2A & G/A & 0.141 & 0.942 & $7.9 \times 10^{-7}$ & 0.78 & $0.770(0.693-0.857)$ \\
\hline
\end{tabular}

The index SNP is defined as the SNP with the smallest $p$ value within a region. The listed gene is our postulated candidate gene near the SNP. Alleles are listed as major/minor (in Europeans). MAF is the frequency of the minor allele in Europeans, and $r^{2}$ is the estimated imputation accuracy. $p_{\text {discovery }}$ and $p_{\text {repl }}$ are the discovery and replication $p$ values, respectively. The OR is the discovery odds ratio per copy of the minor allele (e.g., the A allele of rs72921001 is the allele associated with a lower risk of detecting a soapy taste).

cilantro preference, which are correlated $\left(r^{2} \approx 0.33\right)$. Detection of a soapy taste is reportedly one of the major reasons people seem to dislike cilantro. Despite having over 10,000 more people reporting cilantro preference, we have used soapy-taste detection as our primary phenotype because it is probably influenced by fewer environmental factors. Indeed, we see a stronger effect of rs72921001 on soapy-taste detection than on cilantro preference (OR of 0.81 versus 0.92 ). A GWAS on the replication set gave no genome-wide significant associations. SNPs with $p$ values under $10^{-6}$ for this analysis are shown in Additional file 3.

We find significant differences by sex and ancestral population in soapy-taste detection (Tables 1 and 3). Women are more likely to detect a soapy taste (and to dislike cilantro) (OR for soapy-taste detection 1.36, $p=$ $2.5 \times 10^{-10}$; Table 1). African-Americans, Latinos, East Asians, and South Asians are all significantly less likely to detect a soapy taste compared to Europeans (ORs of $0.676,0.637,0.615$, and 0.270 , respectively, $p<0.003$; see Table 3). Ashkenazi Jews and South Europeans did not show significant differences from Northern Europeans ( $p=0.84$ and 0.65 , respectively). We tested the association between rs72921001 and soapy-taste detection within each population. Aside from the European populations, there was only a significant association in the small South Asian group $(p=0.0078, \mathrm{OR}=0.18,95 \% \mathrm{CI}$ $0.053-0.64)$. This association is in the same direction as the association in Europeans. Note that the GWAS population in Table 1 is a subset of the 'Europe all' population in Table 3, filtered to remove relatives (see the 'Methods' section). While the differences in allele frequency across populations do not explain the differences in soapy-taste detection, our analysis does suggest that this SNP may affect soapy-taste detection in non-European populations as well.

We calculated the heritability for cilantro soapy-taste detection using the GCTA software [27]. We found a low heritability of 0.087 ( $p=0.08,95 \% \mathrm{CI}-0.037$ to 0.211 ). This estimate is a lower bound for the true heritability, as our estimate only takes into account heritability due to SNPs genotyped in this study. While this calculation does not exclude a heritability of zero, the existence of the association with rs72921001 does give a non-zero lower bound on the heritability. Despite the strength of the association of the SNP near OR6A2, it explains only about $0.5 \%$ of the variance in perceiving that cilantro tastes soapy. Our heritability estimate is lower than those given in a recent twin

Table 3 Cilantro soapy-taste by ancestry

\begin{tabular}{|c|c|c|c|c|c|}
\hline Population & Not soapy (\%) & Soapy (\%) & Total & MAF & $p$ value \\
\hline Ashkenazi & $634(85.9 \%)$ & $104(14.1 \%)$ & 738 & 0.355 & 0.56 \\
\hline South Europe & 458 (86.6\%) & 71 (13.4\%) & 529 & 0.335 & 0.25 \\
\hline Europe all & 13,213 (87.0\%) & 1,973 (13.0\%) & 15,186 & 0.373 & $1.23 \times 10^{-8}$ \\
\hline North Europe & 11,794 (87.2\%) & $1,736(12.8 \%)$ & 13,530 & 0.376 & $1.17 \times 10^{-8}$ \\
\hline All & $16,196(87.6 \%)$ & 2,299 (12.4\%) & 18,495 & 0.356 & $3.94 \times 10^{-8}$ \\
\hline African-American & 545 (90.8\%) & 55 (9.2\%) & 600 & 0.224 & 0.87 \\
\hline Latino & 820 (91.3\%) & $78(8.7 \%)$ & 898 & 0.350 & 0.29 \\
\hline East Asia & 424 (91.6\%) & $39(8.4 \%)$ & 463 & 0.283 & 0.22 \\
\hline South Asia & 322 (96.1\%) & $13(3.9 \%)$ & 335 & 0.371 & 0.0078 \\
\hline
\end{tabular}

Number of people detecting a soapy taste by ancestry group, sorted from most to least soapy-taste detection. For reference, we have added the minor allele frequency of rs7107418 in each group. This SNP is a proxy for rs72921001 ( $\left.r^{2}>0.98\right)$, with the minor G allele of rs7107418 corresponding to the minor A allele of rs72921001 (which is associated with less soapy tasting). The $p$ value is the $p$ value of association between soapy-taste and rs7107418 in each group. 
study ( 0.38 for odor and 0.52 for flavor) [7]. This could be due to the differences in phenotypes measured between the two studies, or it could be possible that other genetic factors not detected here could influence cilantro preference. For example, there could be rare variants not typed in this study (possibly in partial linkage disequilibrium with rs72921001) that have a larger effect on cilantro preference. Such rare variants could cause the true heritability of this phenotype to be larger than we have calculated. For example, the heritability of height is estimated to be about 0.8 ; however, the heritability tagged by common SNPs is calculated at about 0.45 [26]. We note that there can be epigenetic modifiers of taste as well, for example, food preferences can even be transmitted to the fetus in utero through the mother's diet [24].

Survey responses, while very efficient for collecting large amounts of data, can only approximately measure the detection and/or perception of the chemicals in cilantro. This has implications for the interpretation of our results. For example, it is possible that the SNP rs72921001 could have a large effect on detection of a specific chemical in cilantro, but that the resulting effect on liking cilantro is much weaker, being modulated by environmental factors. For example, many people might initially dislike cilantro yet later come to appreciate it. This environmental component could also be the reason that our heritability estimates are low. It would thus be interesting to study the genetics of cilantro taste/odor perception in a group without prior exposure to cilantro to reduce the environmental effect, using more direct measures of cilantro perception (i.e., having the subjects actually taste and smell cilantro).

\section{Conclusions}

Through a GWAS, we have shown that a SNP, rs72921001, near a cluster of olfactory receptors is significantly associated with detecting a soapy taste to cilantro. One of the genes near this SNP encodes an olfactory receptor, OR6A2, that detects the aldehydes that may make cilantro smell soapy and thus is a compelling candidate gene for the detection of the cilantro odors that give cilantro its divisive flavor.

\section{Availability of supporting data}

We have shared full summary statistics for all SNPs with $p$ values under $10^{-4}$ in Additional file 2. Due to privacy concerns, under our IRB protocol, we are unable to openly share statistics for all SNPs analyzed in the study.

\section{Methods \\ Subjects}

Participants were drawn from the customer base of 23andMe, Inc., a consumer genetics company. This cohort has been described in detail previously [15,28]. Participants provided informed consent and participated in the research online, under a protocol approved by an external AAHRPP-accredited IRB, Ethical and Independent Review Services (E\&I Review).

\section{Phenotype data collection}

On the 23andMe website, participants contribute information through a combination of research surveys (longer, more formal questionnaires) and research 'snippets' (multiple-choice questions appearing as part of various 23andMe webpages). In this study, participants were asked two questions about cilantro via research snippets:

- 'Does fresh cilantro taste like soap to you?' (Yes/No/I'm not sure)

- 'Do you like the taste of fresh (not dried) cilantro?' (Yes/No/I'm not sure)

Among all 23andMe customers, 18,495 answered the first question (as either yes or no), 29,704 the second, and 15,751 both. Participants also reported their age. Sex and ancestry were determined on the basis of their genetic data. In both the GWAS set and the replication set, all participants were of European ancestry. In either group, no two shared more than $700 \mathrm{cM}$ of DNA identical by descent (IBD, approximately the lower end of sharing between a pair of first cousins). In total, we were left with a set of 14,604 participants who answered the 'soapy' question for GWAS and 11,851 who answered only the taste preference question for a replication set. IBD was calculated using the methods described in [29]; the principal component analysis was performed as in [15]. To determine European and African-American ancestry, we used localancestry methods (as in [30]). Europeans had over 97\% of their genome painted European, and African-Americans had at least $10 \%$ African and at most $10 \%$ Asian ancestry. Other groups were built using ancestry-informative markers trained on a subset of 23andMe customers who reported having four grandparents of a given ancestry.

\section{Genotyping}

Subjects were genotyped on one or more of three chips, two based on the Illumina HumanHap550+ BeadChip and the third based on the Illumina OmniExpress+ BeadChip (San Diego, CA, USA). The platforms contained $586,916,584,942$, and 1,008,948 SNPs. Totals of 291, 5,394 , and 10,184 participants (for the GWAS population) were genotyped on the platforms, respectively. A total of 1,265 individuals were genotyped on multiple chips. For all participants, we imputed genotypes in batches of 8,000-10,000 using Beagle and Minimac [31-33] against the August 2010 release of the 1000 Genomes reference haplotypes [34], as described in [35]. 
A total of $11,914,767$ SNPs were imputed. Of these, $7,356,559$ met our thresholds of 0.001 minor allele frequency, average $r^{2}$ across batches of at least 0.5 , and minimum $r^{2}$ across batches of at least 0.3. The minimum $r^{2}$ requirement was added to filter out SNPs that imputed less well in the batches consisting of the less dense platform. Positions and alleles are given relative to the positive strand of build 37 of the human genome.

\section{Statistical analysis}

For the GWAS, $p$ values were calculated using a likelihood ratio test for the genotype term in the logistic regression model:

$$
Y \sim G+\text { age }+ \text { sex }+\mathrm{pc}_{1}+\mathrm{pc}_{2}+\mathrm{pc}_{3}+\mathrm{pc}_{4}+\mathrm{pc}_{5},
$$

where $Y$ is the vector of phenotypes (coded as $1=$ thinks cilantro tastes soapy or $0=$ does not), $G$ is the vector of genotypes (coded as a dosage 0-2 for the estimated number of minor alleles present), and $\mathrm{pc}_{1}, \ldots, \mathrm{pc}_{5}$ are the projections onto the principal components. The same model was used for the replication, with the phenotype coded as 1 = dislikes cilantro or $0=$ likes. We used the standard cutoff for genome-wide significance of $5 \times 10^{-8}$ to correct for the multiple tests in the GWAS. ORs and $p$ values for the differences in soapy-taste detection between sexes and population were calculated directly, without any covariates. Table 3 uses a proxy SNP for rs72921001, as our imputation was done only in Europeans, so we did not have data for rs72921001 in other populations.

For the heritability calculations, we used the GCTA software [27]. The calculations were done on genotyped SNPs only within a group of 13,628 unrelated Europeans. Unrelated filtering here was done using GCTA to remove individuals with estimated relatedness larger than 0.025 . Thus, this group is slightly different from the GWAS set, as the GWAS set's relatedness filtering was done using IBD. We assumed a prevalence for soapy-taste detection of 0.13 for the transformation of heritability from the 0 1 scale to the liability scale. Otherwise, default options were used. We calculated heritability for autosomal and X chromosome SNPs separately; the estimates were 0.0869 (standard error 0.0634, $p$ value 0.0805) for autosomal SNPs and $2 \times 10^{-6}$ (standard error $0.010753, p$ value 0.5 ) for the $\mathrm{X}$ chromosome.

\section{Additional files}

Additional file 1: Quantile-quantile plot of association with cilantro soapy-taste. Observed $p$ values versus theoretical $p$ values under the nul hypothesis of no association. The genomic control inflation factor for the study was 1.007 and is indicated by the red line; approximate $95 \%$ confidence intervals are given by the blue curves.
Additional file 2: All SNPs with $p<10^{-4}$ for cilantro soapy-taste. Alleles are listed as major/minor. MAF is the frequency of the minor allele in Europeans, and $r^{2}$ is the estimated imputation accuracy. Positions and alleles are given relative to the positive strand of build 37 of the human genome. The gene column shows the position of the SNP in context of the nearest genes. The SNP position is within brackets, and the number of dashes gives approximate $\log _{10}$ distances.

Additional file 3: Index SNPs with $p<\mathbf{1 0}^{-6}$ for cilantro preference in the replication set. Results of a GWAS on cilantro preference in the replication set. Columns are as in Table 2.

\section{Abbreviations}

AAHRPP: Association for the Accreditation of Human Research Protection Programs; BCE: before common era; Cl: confidence interval; GWAS: genome-wide association study; IRB: institutional review board; OR: odds ratio; OR2AG2, OR2AG1, OR6A2, OR1OA5, OR1OA2, OR10A4, OR2D2, OR2D3, members of olfactory receptor gene families 2, 6, and 10; SNP: single-nucleotide polymorphism; TAS1R/TAS2R: taste receptor gene families 1 and 2 .

\section{Competing interests}

The authors of this paper are 23andMe employees and own stock options in the company.

\section{Authors' contributions}

NE, SW, CBD, AKK, JLM, DAH, UF, and JYT conceived and designed the experiments. NE analyzed the data and drafted the manuscript with contributions from all other authors. All authors read and approved the final manuscript.

\section{Acknowledgements}

We thank the customers of 23andMe for participating in this research and all the employees of 23 andMe for contributing to the research.

Received: 14 August 2012 Accepted: 13 November 2012 Published: 29 November 2012

\section{References}

1. Zohary D, Hopf M: Domestication of Plants in the Old World: The Origin and Spread of Cultivated Plants in West Asia, Europe, and the Nile Valley. New York: Oxford University Press; 2000

2. Bostock J, Riley H: The Natural History of Pliny. Volume 4 in Bohn's Classical Library. London: H.G. Bohn; 1855.

3. Faas P: Around the Roman Table. Basingstoke: Palgrave Macmillan; 2002

4. Gerard J: The Herball or General Historie of Plants. Amsterdam: Theatrum Orbis Terrarum; 1974. [1597 ed].

5. Leach $\mathrm{H}$ : Rehabilitating the "stinking herbe": a case study of culinary prejudice. Gastronomica: The J Food Culture 2001, 1(2):10-15.

6. Mauer $\mathrm{L}$, El-Sohemy A: Prevalence of cilantro (Coriandrum sativum) disliking among different ethnocultural groups. Flavour 2012, 1:8.

7. Knaapila A, Hwang LD, Lysenko A, Duke FF, Fesi B, Khoshnevisan A, James RS, Wysocki CJ, Rhyu M, Tordoff MG, Bachmanov AA, Mura E, Nagai H, Reed DR: Genetic analysis of chemosensory traits in human twins. Chem Senses 2012, 37(9):869-881.

8. Cadwallader K, Benitez D, Pojjanapimol S, Suriyaphan O, Singh T: Characteristic aroma components of the cilantro mimics. In Natural Flavors and Fragrances. Volume 908. Edited by Frey C, Rouseff RL. Washington: American Chemical Society; 2005:117-128.

9. Eyres G, Dufour JP, Hallifax G, Sotheeswaran S, Marriott PJ: Identification of character-impact odorants in coriander and wild coriander leaves using gas chromatography-olfactometry (GCO) and comprehensive two-dimensional gas chromatography-time-of-flight mass spectrometry (GC x GC-TOFMS). J Sep Sci 2005, 28(9-10):1061-1074.

10. Li X, Staszewski L, Xu H, Durick K, Zoller M, Adler E: Human receptors for sweet and umami taste. Proc Natl Acad Sci USA 2002, 99(7):4692-4696.

11. Chandrashekar J, Mueller KL, Hoon MA, Adler E, Feng L, Guo W, Zuker CS, Ryba NJ: T2Rs function as bitter taste receptors. Cell 2000, 100(6):703-711. 
12. Reed DR, Knaapila A: Genetics of taste and smell: poisons and pleasures. Prog Mol Biol Trans/ Sci 2010, 94:213-240.

13. Kim UK, Jorgenson E, Coon H, Leppert M, Risch N, Drayna D: Positional cloning of the human quantitative trait locus underlying taste sensitivity to phenylthiocarbamide. Science 2003, 299(5610):1221-1225.

14. Keller A, Zhuang H, Chi Q, Vosshall LB, Matsunami H: Genetic variation in a human odorant receptor alters odour perception. Nature 2007, 449(7161):468-472

15. Eriksson N, Macpherson JM, Tung JY, Hon LS, Naughton B, Saxonov S, Avey L, Wojcicki A, Pe'er I, Mountain J: Web-based, participant-driven studies yield novel genetic associations for common traits. PLOS Genet 2010, 6:e1000993.

16. Kurland MD, Newcomer MB, Peterlin Z, Ryan K, Firestein S, Batista VS Discrimination of saturated aldehydes by the rat 17 olfactory receptor. Biochemistry 2010, 49(30):6302-6304.

17. Araneda $R C$, Kini $A D$, Firestein $S$ : The molecular receptive range of an odorant receptor. Nat Neurosci 2000, 3(12):1248-1255.

18. Krautwurst D, Yau KW, Reed RR: Identification of ligands for olfactory receptors by functional expression of a receptor library. Cell 1998, 95(7):917-926

19. Araneda RC, Peterlin Z, Zhang X, Chesler A, Firestein S: A pharmacological profile of the aldehyde receptor repertoire in rat olfactory epithelium. J Physiol (Lond) 2004, 555(Pt 3):743-756.

20. Buajeeb W, Poomsawat S, Punyasingh J, Sanguansin S: Expression of p16 in oral cancer and premalignant lesions. J Oral Pathol Med 2009, 38:104-108.

21. Luciano M, Lopez LM, de Moor MH, Harris SE, Davies G, Nutile T, Krueger RF, Esko T, Schlessinger D, Toshiko T, Derringer JL, Realo A, Hansell NK, Pergadia ML, Pesonen AK, Sanna S, Terracciano A, Madden PA, Penninx B, Spinhoven P, Hartman CA, Oostra BA, Janssens AC, Eriksson JG, Starr JM, Cannas A, Ferrucci L, Metspalu A, Wright MJ, Heath AC, et al.: Longevity candidate genes and their association with personality traits in the elderly. Am J Med Genet B Neuropsychiatr Genet 2012, 159B(2):192-200.

22. Lopez LM, Harris SE, Luciano M, Liewald D, Davies G, Gow AJ, Tenesa A, Payton A, Ke X, Whalley LJ, Fox H, Haggerty P, Ollier W, Pickles A, Porteous DJ, Horan MA, Pendleton N, Starr JM, Deary IJ: Evolutionary conserved longevity genes and human cognitive abilities in elderly cohorts. Eur J Hum Genet 2012, 20(3):341-347.

23. Manji SS, Williams LH, Miller KA, Ooms LM, Bahlo M, Mitchell CA, Dahl HH: A mutation in synaptojanin 2 causes progressive hearing loss in the ENU-mutagenised mouse strain Mozart. PLOS ONE 2011, 6(3):e17607.

24. Bakalar N: Sensory science: partners in flavour. Nature 2012, 486(7403):4-5.

25. Smith B: Perspective: complexities of flavour. Nature 2012, 486(7403):S6

26. Yang J, Benyamin B, McEvoy BP, Gordon S, Henders AK, Nyholt DR, Madden PA, Heath AC, Martin NG, Montgomery GW, Goddard ME, Visscher PM: Common SNPs explain a large proportion of the heritability for human height. Nat Genet 2010, 42(7):565-569.

27. Yang J, Lee SH, Goddard ME, Visscher PM: GCTA: a tool for genome-wide complex trait analysis. Am J Hum Genet 2011, 88:76-82.

28. Tung JY, Do CB, Hinds DA, Kiefer AK, Macpherson JM, Chowdry AB, Francke U, Naughton BT, Mountain JL, Wojcicki A, Eriksson N: Efficient replication of over $\mathbf{1 8 0}$ genetic associations with self-reported medical data. PLOS ONE 2011, 6:e23473.

29. Henn B, Hon L, Macpherson JM, Eriksson N, Saxonov S, Pe'er I, Mountain $J$ : Cryptic distant relatives are common in both isolated and cosmopolitan genetic samples. PLOS ONE 2012, 7(4):e34267.

30. Eriksson N, Tung JY, Kiefer AK, Hinds DA, Francke U, Mountain JL, Do CB: Novel associations for hypothyroidism include known autoimmune risk loci. PLOS ONE 2012, 7(4):e34442.

31. Browning SR, Browning BL: Rapid and accurate haplotype phasing and missing-data inference for whole-genome association studies by use of localized haplotype clustering. Am J Hum Genet 2007 81:1084-1097.

32. Howie B, Fuchsberger C, Stephens M, Marchini J, Abecasis GR: Fast and accurate genotype imputation in genome-wide association studies through pre-phasing. Nat Genet 2012, 44(8):955-959.

33. Abecasis G, Fuchsberger C: minimac. [http://genome.sph.umich.edu/ wiki/minimac]
34. Altshuler D, Durbin RM, Abecasis GR, Bentley DR, Chakravarti A, Clark AG, Collins FS, De La Vega FM, Donnelly P, Egholm M, Flicek P, Gabriel SB, Gibbs RA, Knoppers BM, Lander ES, Lehrach H, Mardis ER, McVean GA, Nickerson DA, Peltonen L, Schafer AJ, Sherry ST, Wang J, Wilson R, Gibbs RA, Deiros D, Metzker M, Muzny D, Reid J, Wheeler D, et al.: A map of human genome variation from population-scale sequencing. Nature 2010, 467:1061-1073.

35. Eriksson N, Benton GM, Do CB, Kiefer AK, Mountain JL, Hinds DA, Francke $U$, Tung JY: Genetic variants associated with breast size also influence breast cancer risk. BMC Med Genet 2012, 13:53.

doi:10.1186/2044-7248-1-22

Cite this article as: Eriksson et al:: A genetic variant near olfactory receptor genes influences cilantro preference. Flavour 2012 1:22

\section{Submit your next manuscript to BioMed Central and take full advantage of:}

- Convenient online submission

- Thorough peer review

- No space constraints or color figure charges

- Immediate publication on acceptance

- Inclusion in PubMed, CAS, Scopus and Google Scholar

- Research which is freely available for redistribution 